\title{
Cutting a sentinel lymph node into slices is the optimal first step for examination of sentinel lymph nodes in melanoma patients
}

\author{
Christina Mitteldorf ${ }^{1,3,4}$, Hans P Bertsch ${ }^{1,3}$, Antonia Zapf ${ }^{2}$, Christine Neumann ${ }^{1}$ \\ and Lutz Kretschmer ${ }^{1}$ \\ ${ }^{1}$ Department of Dermatology, Venerology and Allergology, Georg August University of Goettingen, Goettingen, \\ Germany and ${ }^{2}$ Department of Medical Statistics, Georg August University of Goettingen, Goettingen, Germany
}

\begin{abstract}
The optimal processing for the pathology of sentinel lymph nodes of patients with melanoma is still a matter of debate. We compared two protocols of sentinel lymph node processing, which were consecutively applied. For the first protocol, the sentinel lymph nodes were cut into 1-2 mm thick slices. From each slice, 12 microtome sections were stained (multiple slices protocol). For the second protocol, which is a modification of the recent European Organisation for Research and Treatment of Cancer protocol, the sentinel lymph nodes were bivalved. Five consecutive series of microtome sections, with gaps of $50 \mu \mathrm{m}$ between them, were prepared from each cut surface (bivalving protocol). H\&E and immunohistochemical staining were integral elements of both protocols. A total of 584 sentinel lymph nodes (1.8 \pm 0.9 per patient) were examined. The percentages of micrometastases (29 versus $27 \%$ ) and of capsular naevi (13 versus $15 \%$ ) detected were very similar for both protocols. As shown by multivariate logistic regression, Breslow thickness $(P=0.003)$ and younger age $(P=0.01)$ correlated with nodal metastasis. The type of histological preparation, ulceration and sex were not significant. The multiple slices protocol produced, on average, 4 paraffin blocks and 46 microtome sections per node. The bivalving protocol constantly produced 2 paraffin blocks and $\mathbf{4 2}$ microtome sections. For technical processing, the multiple slices protocol required, on average, $38 \mathrm{~min}$ per sentinel lymph node, whereas the bivalving protocol required $55 \mathrm{~min}$. Both protocols yielded excellent detection rates with a similar amount of work being required on the part of the pathologist. Compared with the bivalving protocol, the multiple slices protocol was less labor intensive for the technical staff.
\end{abstract}

Modern Pathology (2009) 22, 1622-1627; doi:10.1038/modpathol.2009.137; published online 2 October 2009

Keywords: melanoma; sentinel lymph node; processing for pathology; micrometastasis; nodal nevus; sensitivity

A sentinel lymph node is defined as the first lymph node on the lymphatic drainage pathway from the primary tumor site. In cutaneous melanoma, the pathological status of the sentinel lymph node has been established as the most important prognostic factor for recurrence and survival. For patients with micrometastasis, the impact of nodal tumor load and different metastasising patterns within the sentinel lymph node are of particular interest. Data

Correspondence: Dr C Mitteldorf, MD, Department of Dermatology, Venerology and Allergology, Klinikum Hildesheim GmbH, Weinberg 1, D-31134 Hildesheim, Germany.

E-mail: christina.mitteldorf@gmx.de

${ }^{3}$ These authors contributed equally to this work.

${ }^{4}$ Current address: Department of Dermatology, Venerology and Allergology, Klinikum Hildesheim GmbH, Weinberg 1, D-31134 Hildesheim, Germany.

Received 23 June 2009; revised 13 August 2009; accepted 14 August 2009; published online 2 October 2009 suggesting that these patients may benefit from early excision of their nodal metastases are available..$^{1,2}$ The presence or absence of micrometastasis in the sentinel lymph node is critical for decision making with regard to complete lymphadenectomy and stratification for adjuvant therapy trials.

These important issues require sufficiently sensitive and reliable methods for the diagnosis of sentinel lymph node metastasis. In clinical practice, the detection rates reported varied widely, between 14 and $33.8 \% .^{3-10}$ This has to be attributed, at least to some extent, to differences in the processing of the sentinel lymph nodes for pathology. To provide comparability between different institutions, standardised pathological protocols are required. However, even with extended serial sectioning and immunohistochemical staining, small metastases or single melanoma cells may easily be missed. The reason is that complete serial sectioning of the 
entire sentinel lymph node is almost impossible. As a consequence, any type of pathological processing can only provide a compromise between sufficiently high sensitivity and acceptable expenditure.

Two principally different types of protocols have been proposed as being practicable and are widely used. The first protocol is based on cutting the sentinel lymph node into $1-3 \mathrm{~mm}$ thick slices. ${ }^{11} \mathrm{~A}$ certain number of microtome sections from each slice is stained by H\&E and immunohistochemistry.

For the second type of histological processing, the sentinel lymph node is bisected through its longest meridian and serial sections are taken commencing from both cut surfaces (bivalving protocol). ${ }^{12}$ Cook et $a l^{6}$ improved this bivalving protocol by adding six additional series of microtome sections, with gaps of $50 \mu \mathrm{m}$ between them. Excellent detection rates of up to $33.8 \%$ have been achieved using this protocol, which is currently recommended by the European Organisation for Research and Treatment of Cancer (EORTC). ${ }^{4,6}$

We have consecutively applied both types of histological processing at our institute. In this study, we compare a multiple slices protocol with a bivalving protocol in terms of sensitivity and the workload placed on the pathology laboratory.

\section{Patients and methods}

From November 1997 to December 2006, 299 patients with primary malignant melanomas $\geq 1 \mathrm{~mm}$ thick received a sentinel lymph node biopsy at the Department of Dermatology of the University of Goettingen. Before surgery, metastases were excluded by physical examination, ultrasound Bscans of regional lymph nodes and abdomen, as well as X-rays of the chest.

The standard treatment for a primary melanoma is wide local excision with adequate safety margins, depending on tumor thickness. Dynamic and static lymphoscintigraphy were performed using 99mTclabelled human albumin. During sentinel lymph node biopsy, blue dye and a hand-held $\gamma$-probe were used to localise the sentinel lymph nodes. Lymph nodes that stained blue and had blue afferent lymph channels were generally defined as sentinel lymph nodes. Radioactive lymph node(s) that had first appeared during dynamic lymphoscintigraphy or for which there was lymphoscintigraphic evidence of an own afferent lymphatic vessel were also defined as sentinel lymph node(s). The primary tumor characteristics and the results of sentinel lymph node assessment were prospectively entered into an electronic database.

\section{Processing and Histological Evaluation of Sentinel Lymph Nodes}

Multiple Slices Protocol

From November 1997 to December 2003, we used a multiple slices protocol for histological processing of sentinel lymph nodes. The formalin-fixed sentinel lymph nodes were cut into 1-2 mm thick tissue slices, which were separately embedded in paraffin. At least 12 consecutive paraffin sections were cut from each block and stained in the following order: 1 . S-100; 2. HMB-45; 3. MART-1; 4-9. H\&E; 10. S-100; 11. HMB-45; 12. MART-1.

\section{Bivalving Protocol}

In January 2004, we introduced a new protocol according to the recent EORTC guidelines. For this protocol, the nodes were bisected through their hilum at the longest meridian and embedded in paraffin. Four series of paraffin sections were prepared from the cut surfaces of both halves and stained with $\mathrm{H} \& \mathrm{E}$ and immunohistochemical markers (such as S-100, MART-1, HMB-45, pan-melanoma-plus), with gaps of $50 \mu \mathrm{m}$ between them. This protocol produced 42 slides from each sentinel lymph node. As a modification of the original EORTC protocol, we introduced the MART-1 antibody and increased the number of HMB-45-stained sections. Owing to the increased number of slides, we performed only four instead of five series of microtome sections for evaluating the pathology. Details of the staining regimen are shown in Table 1.

\section{Immunohistochemistry}

Immunohistochemical staining was performed by applying the streptavidin-biotin complex method with alkaline phosphatase as the labelling enzyme and fast red chromogen as the substrate (Dako, Germany, detection kit K5005). The following antibodies were used: S-100 (Dako, clone: S-100, dilution: 1:3000), HMB-45 (Dako, clone: HMB45, dilution: 1:200), MART-1 (Zymed, USA, clone: A 103/M2-7C10/M2-9E3, dilution: 1:200) and PanMelanoma Cocktail (Biocare Medical, USA, clone: HMB45 + M2-7C10 + M2-9E3 + T311, dilution: 1:300) stained by an auto-immunostainer (Immunostar 80, Shandon Varistain 24-4, Germany).

\section{Analysis of Labor Input}

To objectively quantify the amount of work required of the pathologist, we measured the mean number of slides for evaluating the pathology according to the protocol applied. To evaluate the time taken for histological processing requiring an assistant medical technician, we exemplarily conducted a study on 10 lymph nodes from a completion lymph node dissection. These lymph nodes were precisely bivalved, so that two equal halves with nearly equal consistency and tissue volume were produced. One half was processed by the multiple slices protocol and the other half by the bivalving protocol. The technical work was carried out by an experienced technical assistant. The time required for each type of processing was precisely 
Table 1 Histological processing of the bisecting protocol ( ${ }^{*}$ p-Mel $=$ pan-melanoma-plus) compared with the EORTC protocol $^{6}$

\begin{tabular}{|c|c|c|c|}
\hline \multicolumn{2}{|c|}{ Bivalving protocol (BP) } & \multicolumn{2}{|c|}{ EORTC protocol } \\
\hline 1. & $\mathrm{H} \& \mathrm{E}$ & 1. & $\mathrm{H} \& \mathrm{E}$ \\
\hline 2. & S-100 & 2. & S-100 \\
\hline 3. & HMB-45 & - & - \\
\hline 4. & MART-1 & - & - \\
\hline 5. & Spare & 3. & Spare \\
\hline 6. & Spare & - & - \\
\hline \multicolumn{2}{|l|}{ 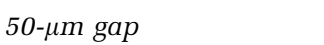 } & \multicolumn{2}{|l|}{ 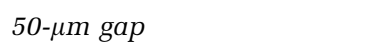 } \\
\hline 7. & $\mathrm{HE}$ & 4. & $\mathrm{H} \& \mathrm{E}$ \\
\hline 8. & S-100 & 5. & S-100 \\
\hline 9. & HMB-45 & 6. & HMB-45 \\
\hline 10. & MART-1 & - & - \\
\hline 11. & p-Mel & 7. & p-Mel \\
\hline 12. & Spare & 8. & Spare \\
\hline 13. & Spare & 9. & Spare \\
\hline \multicolumn{2}{|l|}{ 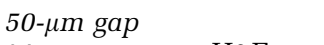 } & \multicolumn{2}{|l|}{$50-\mu \mathrm{m}$ gap } \\
\hline 14. & $\mathrm{H} \& \mathrm{E}$ & 10. & $\mathrm{H} \& \mathrm{E}$ \\
\hline 15. & S-100 & 11. & S-100 \\
\hline 16. & HMB-45 & - & - \\
\hline 17. & MART-1 & - & - \\
\hline 18. & Spare & 12. & Spare \\
\hline 19. & Spare & - & - \\
\hline \multicolumn{2}{|l|}{ 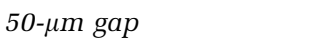 } & \multicolumn{2}{|l|}{$50-\mu \mathrm{m}$ gap } \\
\hline 20. & H\&E & 13. & $\mathrm{H} \& \mathrm{E}$ \\
\hline 21. & S-100 & 14. & S-100 \\
\hline 22. & HMB-45 & - & - \\
\hline 23. & MART-1 & - & - \\
\hline 24. & Spare & 15. & Spare \\
\hline 25. & Spare & - & - \\
\hline \multicolumn{2}{|l|}{ 50- $\mu$ gар } & \multicolumn{2}{|l|}{ 50- $\mu$ m gap } \\
\hline 26. & $\mathrm{H} \& \mathrm{E}$ & 16. & $\mathrm{H} \& \mathrm{E}$ \\
\hline 27. & S-100 & 17. & S-100 \\
\hline 28. & HMB-45 & - & - \\
\hline \multirow[t]{4}{*}{29.} & MART-1 & 一 & - \\
\hline & & $\begin{array}{l}18 . \\
50-\mu m \text { gap }\end{array}$ & Spare \\
\hline & & 19. & $\mathrm{H} \& \mathrm{E}$ \\
\hline & & 20. & S-100 \\
\hline
\end{tabular}

measured and then extrapolated to the time required per sentinel lymph node.

\section{Sentinel Lymph Node Assessment}

All sections were examined and/or reviewed by two experienced dermato-pathologists. In the event of diagnostic difficulties, external experts were consulted. Melanoma and nevus cells had to be differentiated by morphological, immunophenotypical and localisation criteria. We use the term 'sentinel lymph node metastasis' as equivalent to the presence of three or more adjacent melanoma cells in a sentinel lymph node. The maximum depth of invasion of the metastases into the sentinel lymph node was measured from the nodal capsule, according to the S-classification. ${ }^{13}$

\section{Statistical Methods}

For statistical analyses, we used SAS 9.1 (SAS Institute, Cary, NC, USA). Testing of means for factors that might influence the probability of micrometastasis was performed using the WilcoxonMann-Whitney test. The Fisher exact test was used to compare the proportion of positive sentinel lymph nodes obtained with both protocols. Logistic regression was used to evaluate the relative power of the various factors predicting the likelihood of nodal metastasis. Significance was determined at the $P<0.05$ level.

\section{Results}

\section{Patient Characteristics}

Of the 299 patients included in this study, 158 $(53 \%)$ were men. The median age was 63 years (16-89 years), the mean Breslow thickness was $2.77 \mathrm{~mm}$ (median, range $1.00-16.00 \mathrm{~mm}$ ) and the percentage of ulceration was $33 \%$. A total of 584 sentinel lymph nodes were examined (multiple slice protocol $n=305$; bivalving protocol $n=279$ ). Micrometastasis to a sentinel lymph node was diagnosed in $28 \%$ of the patients. The median invasion depth of the metastasis into the lymph node according to the study by Starz et $a l^{13}$ was $0.74 \mathrm{~mm}$ $(0.01-6.30 \mathrm{~mm})$. The mean number of sentinel lymph nodes excised per patient was $1.8 \pm 0.9$ (median: 2 (1-5)). Table 2 shows the patient characteristics in relation to the protocol applied. Breslow thickness, ulceration and nodal tumor burden according to the S-classification were well matched between the two groups studied. Unexpectedly, patients whose sentinel lymph nodes were processed according to the bivalving protocol were, on average, 6 years older.

\section{Sensitivity According to the Protocol Applied}

The percentages of diagnosed capsular naevi per patient were 13 (multiple slice protocol) and 15\% (bivalving protocol), and the percentages of nodal micrometastases were 29 (multiple slice protocol) and $27 \%$ (bivalving protocol). These differences were not statistically significant.

\section{Risk Factors Predicting Micrometastasis to a Sentinel Lymph Node}

Univariate logistic regression showed that increasing Breslow thickness $(P=0.003)$ and younger age $(P=0.01)$ were significantly correlated with micrometastasis to the sentinel lymph node. The following features were not significant: ulceration, sex and the type of histological protocol applied. Using multivariate logistic regression, Breslow $(P=0.003)$ and age $(P=0.01)$ remained significant. Importantly, there was no difference in the protocol applied. The full model is shown in Table 3. 
Table 2 Patient characteristics according to the protocol used

\begin{tabular}{lccc}
\hline & $\begin{array}{c}\text { Multiple slices } \\
\text { protocol, } \mathrm{N}=171\end{array}$ & $\begin{array}{c}\text { Bivalving } \\
\text { protocol, } \\
\mathrm{N}=128\end{array}$ & $\begin{array}{c}\text { P-value } \\
\text { (U-test) }\end{array}$ \\
\hline Breslow & Mean: $-2.80 \mathrm{~mm}$ & $2.74 \mathrm{~mm}$ & 0.12 \\
Ulceration & $56(33 \%)$ & $42(33 \%)$ & 0.98 \\
Age & Mean:-57 mm & 63 & 0.002 \\
Male sex & $88(51 \%)$ & $70(55 \%)$ & 0.58 \\
Capsular naevi & $20(13 \%)$ & $19(15 \%)$ & 0.67 \\
SLN metastasis $(\%)$ & $50(29 \%)$ & $35(27 \%)$ & 0.72 \\
Invasion depth & Mean: $-1.50 \mathrm{~mm}$ & $1.14 \mathrm{~mm}$ & 0.31 \\
(according to Starz & & &
\end{tabular}

Table 3 Factors influencing the sensitivity for the detection of SLN metastasis (*univariate logistic regression, ${ }^{* *}$ multivariate logistic regression)

\begin{tabular}{lcc}
\hline & P-value* & P-value \\
\hline Breslow index & 0.003 & 0.01 \\
Age & 0.01 & 0.002 \\
Ulceration & 0.06 & 0.38 \\
Sex & 0.19 & 0.37 \\
Protocol (MSP versus BP) & 0.72 & 0.41
\end{tabular}

\section{Labor Input of the Pathologist}

For the multiple slice protocol, the median number of cut levels (slices) was 4 (range: 1-17). Thus, on average, four paraffin blocks were produced. An average of 46 (range: 12-204) microtome sections were cut and stained.

For the bivalving protocol, only two paraffin blocks per sentinel lymph node were produced. This resulted in a constant number of 42 microtome sections for staining. Consequently 46 versus 42 slides had to be evaluated per sentinel lymph node. As macroscopic cutting of the surgical specimens takes only a few seconds, the labor input for the pathologist was not significantly influenced by the type of histological procedure.

\section{Labor Input of the Technical Staff}

The time expenditure for embedding, clamping in the microtome, cutting and producing the slices is shown in Table 3. Bivalving protocol required, on average, $55 \mathrm{~min}$ for technical processing of one sentinel lymph node, whereas the multiple slice protocol required only $38 \mathrm{~min}$. The most timeconsuming step in the bivalving protocol was creating the 'gaps' between two levels of serial sectioning and staining. The next level for pathological evaluation was reached by performing $3-\mu \mathrm{m}$ thick microtome sections, which necessitated breaks after every 4-10 sections for cooling down and repeated clamping of the paraffin blocks (Table 4).
Table 4 A comparison of the amount of work involved in technical processing resulting from the two protocols

\begin{tabular}{lcc}
\hline & $\begin{array}{c}\text { Mean measured time per SLN } \\
\text { in minutes }\end{array}$ \\
\cline { 2 - 3 } & MSP & $B P$ \\
\hline $\begin{array}{l}\text { Embedding } \\
\begin{array}{l}\text { Clamping in the microtome and } \\
\text { first cut }\end{array}\end{array}$ & 12 & 6 \\
$\begin{array}{l}\text { Cutting and slicing } \\
\text { Total }\end{array}$ & 2 & 1 \\
& 24 & 48 \\
& 38 & 55
\end{tabular}

\section{Discussion}

This study evaluated the sensitivity of two widely used protocols, both comprising extended serial sectioning, H\&E and immunohistochemistry, referred to in this study as 'multiple slices protocol' and 'bivalving protocol.' These terms reflect the first step in the pathological work-up, in which a sentinel lymph node is either bivalved through its hilum or cut into $1-2 \mathrm{~mm}$ thick slices.

The detection rate of micrometastases achieved using our multiple slice protocol was $29 \%$ (detection rate of nodal naevi: $13 \%$ ). With our bivalving protocol, which is a modification of the present EORTC protocol, we achieved a micrometastase detection rate of $27 \%$ (detection rate of nodal naevi: $15 \%)$. On the basis of multivariate logistic regression, the type of pathology processing did not influence the micrometastases detection rate, whereas the probability of sentinel lymph node metastasis increased with thicker primary tumors and younger age. The latter finding seems to be surprising, at first view, but it has been confirmed by others. ${ }^{14-16}$

Unfortunately, patients with sentinel lymph nodes processed by the bivalving protocol were, on average, 6 years older compared with those whose sentinel lymph nodes were analysed according to the multiple slice protocol method, so that the sensitivity of the bivalving protocol is possibly underestimated in this study. The mean Breslow thickness and the mean invasion depth of the metastasis into the sentinel lymph node, ${ }^{13}$ which is a surrogate for the nodal tumor burden, did not differ significantly between the two compared time periods with different pathology protocols.

Sentinel lymph node micrometastasis usually starts from the subcapsular sinuses through an afferent capsular lymph vessel. Later on, the tumor spread continues from the cortical parenchyma to deeper zones of the lymph node, frequently following the medullary sinuses. Two strategies to detect a maximum of micrometastases are available. Cochran $^{12}$ and others ${ }^{5}$ have argued that the majority of micrometastases is located in a central plane through the hilum and the longest dimension of the lymph node and have postulated accurate bisection 
of the sentinel lymph node. Cochran's bivalving protocol, ${ }^{12}$ constantly producing 16 sections per sentinel lymph node, has yielded relatively low detection rates of $18 \%$, on average. ${ }^{17-22}$ Several authors have claimed that a certain percentage of micrometastases may be missed by sampling only the central plane of the sentinel lymph node. ${ }^{13,17,23}$ Consequently, they have preferred to perform multiple parallel slicing of the node, not necessarily through the hilum, and examining a series of sections from each slice.

Both approaches have their strengths and weaknesses. In our experience, bisecting a sentinel lymph node exactly through its hilum is an ideal, which is hardly ever achieved in practice. The recent EORTC protocol, an extended bivalving protocol, is designed to nearly completely sample an $\sim 0.6-\mathrm{mm}$ thick slice from the central plane of the sentinel lymph node. With the EORTC protocol, a standardised number of 40 microtome slices per sentinel lymph node is stained by $\mathrm{H} \& \mathrm{E}$ and immunohistochemistry. Excellent detection rates of up to $33.8 \%$ were achieved. ${ }^{4,6}$ However, as reported by others, ${ }^{24}$ we experienced difficulties in detecting single cells or small metastases without using the MART-1 antibody. MART-1 was therefore added at five different positions in our bivalving protocol (see Table 1). MART-1 exhibited the same sensitivity as did S-100, but it is more specific in sentinel lymph nodes. ${ }^{23}$ Furthermore, we increased the number of HMB-45-stained sections, to better differentiate nevus cell inclusions from melanoma cells. ${ }^{23-26}$ In contrast to S-100, HMB-45 is more specific for melanocytes, but $18-35 \%$ of the melanoma metastasis are HMB-45 negative. ${ }^{5,25,26}$ Owing to the increased number of slides, up to 42 , we analysed only 4 instead of 5 series of microtome sections, thereby reaching a depth of $\sim 287 \mu \mathrm{m}$ from the cut surface of the sentinel lymph node (instead of $310 \mu \mathrm{m}$ according to the EORTC protocol). The 'gaps' of $50 \mu \mathrm{m}$ between the series of stained sections still allowed detection of most micrometastases, which are usually larger than $50 \mu \mathrm{m}$. These gaps are achieved by performing multiple unstained microtome sections, which is the most time-consuming step. A further disadvantage of these gaps is the irreversible loss of these areas for later histological examination. Therefore, 6-8 'spare' sections should be introduced at different positions, which have to be stored under special conditions to prevent their destruction.

In contrast, a multiple slice protocol does not need 'spare' sections. Most likely, with a multiple slice protocol as well, a roughly central plane of the sentinel lymph node is sampled, although less intensively. The thickness of the nodal slices (1$3 \mathrm{~mm}$ ) is not well standardised. Our multiple slice protocol produced on average 4 slices and 12-204 histological slides, depending on the size of the respective sentinel lymph nodes. Compared with our bivalving protocol, our multiple slice protocol produced fewer slides for smaller sentinel lymph nodes, but more slides for larger sentinel lymph nodes, requiring five or more nodal slices. Therefore, the bivalving protocol theoretically provides a more intensive sampling of small sentinel lymph nodes, whereas the multiple slice protocol theoretically provides more intensive examination of larger sentinel lymph nodes. In our hands, the sensitivity for an average sentinel lymph node was nearly identical under both protocols. As a consequence, the expenditure of labor becomes important. Both the multiple slice protocol and the bivalving protocol produced similar numbers of histological slides (average 46 versus 42), suggesting that the amount of work remained the same for the pathologist. Surprisingly, the time expenditure for embedding, clamping in the microtome, cutting and producing the slices was higher for the bivalving protocol. The bivalving protocol required, on average, $55 \mathrm{~min}$ per sentinel lymph node, whereas the multiple slice protocol required only $38 \mathrm{~min}$. For an average patient with two sentinel lymph nodes, the multiple slice protocol saves approximately half an hour for technical processing.

In conclusion, both protocols yield excellent detection rates with similar labor input by the pathologist. Compared with the bivalving protocol, the multiple slice protocol was significantly less labor intensive for the technical staff; the loss of a patient's material must also be taken into account for further investigations. Consequently, we returned to our formerly used multiple slice protocol.

\section{Acknowledgements}

We did not obtain any funding and have no relevant financial interest in this article. We thank Roswitha Habel, Ute Kant and Desirée Wolters for the excellent technical assistance. We also thank Sabine Steylaers for her brilliant support with data collection and administration.

\section{Disclosure/conflict of interest}

The authors declare no conflict of interest.

\section{References}

1 Kretschmer L, Hilgers R, Möhrle M, et al. Patients with lymphatic metastasis of cutaneous malignant melanoma benefit from sentinel lymphonodectomy and early excision of their nodal disease. Eur J Cancer 2004;40:212-218.

2 Morton DL, Thompson JF, Cochran AJ, et al. Sentinelnode biopsy or nodal observation in melanoma. N Engl J Med 2006;355:1307-1317.

3 Kretschmer L, Beckmann I, Thoms KM, et al. Sentinel lymphonodectomy does not increase the risk of loco- 
regional cutaneous metastases of malignant melanomas. Eur J Cancer 2005;41:531-538.

4 van Akkooi ACJ, de Wilt JHW, Verhoef C, et al. High positive sentinel node identification rate by EORTC melanoma group protocol. Prognostic indicators of metastatic patterns after sentinel node biopsy in melanoma. Eur J Cancer 2006;42:372-380.

5 Abrahamsen HN, Hamilton-Dutoit SJ, Larsen J, et al. Sentinel lymph nodes in malignant melanoma: extended histopathologic evaluation improves diagnostic precision. Cancer 2004;100:1683-1691.

6 Cook MG, Green MA, Anderson B, et al. The development of optimal pathological assessment of sentinel lymph nodes for melanoma. J Pathol 2003;200: 314-319.

7 Carlson GW, Murray DR, Lyles RH, et al. The amount of metastatic melanoma in a sentinel lymph node: does it have prognostic significance? Ann Surg Oncol 2003;10:575-581.

8 Estourgie SH, Nieweg OE, Valdés Olmos RA, et al. Review and evaluation of sentinel node procedures in 250 melanoma patients with a median follow up of 6 years. Ann Surg Oncol 2003;10:681-688.

9 Gershenwald JE, Thompson W, Mansfield PF, et al. Multi-institutional melanoma lymphatic mapping experience: the prognostic value of sentinel lymph node status in 612 stage I or II melanoma patients. J Clin Oncol 1999;17:976-983.

10 Vuylsteke RJ, van Leeuwen PA, Statius Muller MG, et al. Clinical outcome of stage I/II melanoma patients after selective sentinel lymph node dissection: long-term follow-up results. J Clin Oncol 2003;21:1057-1065.

11 Cascinelli N, Belli F, Santinami M, et al. Sentinel lymph node biopsy in cutaneous melanoma: the WHO Melanoma Program experience. Ann Surg Oncol 2000;7:469-474.

12 Cochran AJ. Surgical pathology remains pivotal in the evaluation of 'Sentinel' lymph nodes. Am J Surg Pathol 1999;23:1169-1172.

13 Starz H, Balda BR, Krämer KU, et al. A micromorphometry-based concept for routine classification of sentinel lymph node metastases and its clinical relevance for patients with melanoma. Cancer 2001;91:2110-2121.

14 McMasters KM, Wong SL, Edwards MJ, et al. Factors that predict the presence of sentinel lymph node metastasis in patients with melanoma. Surgery 2001; 130:151-156.
15 Sondak VK, Taylor JM, Sabel MS, et al. Mitotic rate and younger age are predictors of sentinel lymph node positivity: lessons learned from the generation of a probabilistic model. Ann Surg Oncol 2004;11: 247-258.

16 Roaten JB, Partrick DA, Bensard D, et al. Survival in sentinel lymph node-positive pediatric melanoma. J Pediatr Surg 2005;40:988-992.

17 Bieligk SC, Ghossein R, Bhattacharya S, et al. Detection of tyrosinase mRNA by reverse transcriptionpolymerase chain reaction in melanoma sentinel nodes. Ann Surg Oncol 1999;6:232-240.

18 Blaheta H, Ellwanger U, Schittek B, et al. Examination of regional lymph nodes by sentinel node biopsy and molecular analysis provides new staging facilities in primary cutaneous melanoma. J Invest Dermatol 2000;114:637-642.

19 Gogel BM, Kuhn JA, Ferry KM, et al. Sentinel lymph node biopsy for melanoma. Am J Surg 1998;176: 544-547.

20 Shivers SC, Wang X, Li W, et al. Molecular staging of malignant melanoma: correlation with clinical outcome. JAMA 1998;280:1410-1415.

21 van der Velde-Zimmermann D, Schipper ME, de Weger RA, et al. Sentinel node biopsies in melanoma patients: a protocol for accurate, efficient, and cost-effective analysis by preselection for immunohistochemistry on the basis of Tyr-PCR. Ann Surg Oncol 2000;7:51-54.

22 Statius Muller MG, van Leeuwen PAM, de Lange-de Klerk ES, et al. The sentinel lymph node status is an important factor for predicting clinical outcome in patients with stage I or II cutaneous melanoma. Cancer 2001;91:2401-2408.

23 Ruiter DJ, Spatz A, van den Oord JJ, et al. Pathologic staging of melanoma. Semin Oncol 2002;29:370-381.

24 Satzger I, Völker B, Meier A, et al. Prognostic significance of isolated HMB45 or Melan A positive cells in melanoma sentinel lymph nodes. Am J Surg Pathol 2007;31:1175-1180.

25 Shidham VB, Qi DY, Acker S, et al. Evaluation of micrometastases in sentinel lymph nodes of cutaneous melanoma: higher diagnostic accuracy with Melan-A and MART-1 compared with S-100 protein and HMB45. Am J Surg Pathol 2001;25:1039-1046.

$26 \mathrm{Yu} \mathrm{LL}$, Flotte TJ, Tanabe KK, et al. Detection of microscopic melanoma metastases in sentinel lymph nodes. Cancer 1999;86:617-627. 